Citation: Frère, J., Sanchez, H., Vanhaesebrouck, R., \& Cassirame, J. (2019, February 7). Effect of simplifying the body model to compute the energy parameters in pole vaulting. https://doi.org/10.31236/osf.io/hv36w

\title{
Effect of simplifying the body model to compute the energy parameters in pole vaulting
}

\author{
$\underline{\text { Julien FRÈRE }}^{1}, \underline{\text { Hervé SANCHEZ }^{4}}$, Romain VANHAESEBROUCK ${ }^{2}$, Johan \\ CASSIRAME $^{2,3,4}$
}

1. Université de Lorraine, DevAH, F-54000 Nancy, France.

2. Université de Franche-Comté, EA 4660 Culture Sport Health Society, EPSI, F-25000 Besançon, France

3. Université de Reims Champagne-Ardenne, EA 7507 Laboratoire Performance, Santé, Métrologie, Société, F-51100 Reims, France.

4. Fédération Française d'Athlétisme, 33 avenue Pierre-de-Coubertin, F-75000 Paris, France

\section{Correspondence:}

Julien FRERE, PhD,

Université de Lorraine

EA 3450 DevAH - Faculté des Sciences du Sport

30 rue du Jardin Botanique, CS 30156

54603 Villers-lès-Nancy Cedex, FRANCE

Phone : +33(0)3 72746739

Fax : +33(0)372746712

E-mail: julien.frere@univ-lorraine.fr

All co-authors approved the content of this submission and agree with the fact that it is shared as a pre-print.

DOI: $\underline{10.31236 / \text { osf.io/hv36w }}$ 


\begin{abstract}
This study compared changes in the energy time-profiles and related parameters in pole vaulting using three body models. Two-dimensional kinematics were collected from 20 trials (5.20-6.01 $\mathrm{m})$ performed by 10 athletes during a competition. The linear and angular kinetic, potential, and mechanical energy time-profiles were obtained from three pole vaulter models composed of 12,5 , and 3 segments $\left(\mathrm{M}_{12}, \mathrm{M}_{5}\right.$, and $\mathrm{M}_{3}$, respectively). The energy values of $\mathrm{M}_{5}$, compared to those of $\mathrm{M}_{3}$, were more similar to $\mathrm{M}_{12}$ and appeared to be a good compromise between the decreased number of digitized points and the reliability of the energetic outcomes in pole vaulting.
\end{abstract}

Keywords: kinematics, motion capture, sport performance.

\title{
Introduction
}

In pole vaulting, athlete benefit from the elastic properties of the pole to gain in total mechanical energy during the pole support phase (Dillman and Nelson 1968; Hay 1971). The total mechanical energy is lower at take-off, with a high proportion of translational kinetic energy, than at the crossbar, with a high proportion of potential energy (Dillman and Nelson 1968; Hay 1971; Gros and Kunkel 1990; Frère et al. 2010). Even if a high amount of the athlete's horizontal velocity at take-off remains a major performance criterion (Adamczewski and Perlt 1997), maximizing this energy gain during the pole support phase is of interest because the total mechanical energy at the crossbar highly correlates with performance (Arampatzis et al. 1999).

Experimental (Arampatzis et al. 2004; Frère et al. 2012) and simulation studies (Hubbard 1980; Ekevad and Lundberg 1995, 1997) have both shown that this energy gain is obtained by means of muscular work. Though experimental studies have used full body models to compute the energetic time-profiles (kinetic, potential, and mechanical), simulation studies have used a simplified body model to compute the internal work performed by the athlete to interact with the pole. For example, using a three-segments model (upper limb, trunk, and lower limb) able to generate torque around the shoulder, hip, and wrist joints, Hubbard (1980) showed that the work done by the shoulders contributed the most to the mechanical energy gain during the pole vault. Surface electromyographic records corroborated the result, with an increase in latissimus dorsi muscle activity during the swing-to-inversion phase (Frère et al. 2012).

The use of anatomical markers and anthropometric tables in a full-body model of the athlete allows calculation of the kinematics and dynamics of each body segment and the center of mass (CoM) of the model. In the case of pole vaulting, the complexity of the computation approach had little effect on the energy parameters of interest (i.e., at take-off, at time of maximal pole bending, when crossing the bar). Schade et al. (2000) found differences of less than $1 \%$ when comparing three-dimensional and two-dimensional approaches to energy computation, whereas differences of up to $4 \%$ were found when comparing energy parameters obtained from the kinematics of all body segments (total energy) and the parameters obtained solely from the kinematics of CoM (CoM energy). These results indicate that the movements 
performed out of the main plane of motion (i.e., sagittal) and those performed around the CoM have negligible effects on the energy time-profiles and parameters during pole vaulting.

These previous results are relevant, especially for the purposes of understanding performance and training, as the energy time-profiles and their related parameters were reproducible (Schade et al. 2006). Thus, the simplification of data acquisition allows embedding a scientific follow-up to help coaches define technical goals in their training programs. In addition, reducing the delay between trial performance and visualization of the energy profile (which can be seen as feedback promoting an external focus) may contribute to enhanced skill learning (Wulf et al. 2001; Wulf, Chiviacowsky, et al. 2010), performance, and movement efficiency (Marchant et al. 2009; Wulf, Dufek, et al. 2010; Abdollahipour et al. 2015). However, even if an economical approach can be considered in pole vaulting, the use of a full body model is still necessary to compute the kinematics of the CoM. This is especially true given that recordings during official competitions do not always meet the required setup (passive or active anatomical body markers, optimal cameras position, uniform background, etc.) to automatically track the body motion, even in two dimensions. Therefore, it is of interest to assess the effect of simplifying the body model of the pole vaulter on these energy values, by reducing the number of markers to digitize per frame.

The aim of the present study was to compare the energy time-profiles (kinetic, potential, and mechanical) and parameters from three body models of the pole vaulter. The effect of the calculation approach (total vs. CoM energy) was also investigated. We expected to find similar results among the models and calculation approaches.

\section{Methods}

\section{Participants}

Data were collected during the men's pole vault final at the 2015 Indoor Track and Field National Elite French Championships in Aubière, France. Ten athletes participated in this competition (age: 22-32 years; weight: $65-82 \mathrm{~kg}$; height: $171-189 \mathrm{~cm}$; personal best at the time of the competition: 5.44-6.16 m, corresponding to $88-100 \%$ of the world record). Taking into account the rises of the crossbar and the way the competition ran, 20 valid jumps were retained for analysis. The performances were distributed as follows: $5.20 \mathrm{~m}(n=3), 5.35 \mathrm{~m}(n$ $=6), 5.50 \mathrm{~m}(n=5), 5.60 \mathrm{~m}(n=2), 5.66 \mathrm{~m}(n=2), 5.94 \mathrm{~m}(n=1)$, and $6.01 \mathrm{~m}(n=1)$. A third valid jump at $5.60 \mathrm{~m}$ was not selected for further analysis due to a technical issue. All athletes were right-handed and free of injury when data were collected. They were informed about the measurements during the competition and provided written consent. This study was conducted in accordance with the recommendations of the Declaration of Helsinki.

\section{Data collection}

The vaults were captured by one high-speed video camera $(100 \mathrm{~Hz}$, Sony HDR-XR160, Tokyo, Japan) positioned above the galleries, perpendicular to the runway on the left side, approximately $25 \mathrm{~m}$ from the midline of the runway, $5 \mathrm{~m}$ above the ground level, and $2.5 \mathrm{~m}$ in front of the end of the planting box. This position allowed the camera to capture the last two strides of the run-up and the full athlete-pole interaction phase up to the bar clearance within 
the center area of the field of the camera $(1920 \times 1080$ pixels $)$. The accuracy of the determination of the spatial coordinates was $\pm 1.2 \mathrm{~cm}$, which is in line with previous recordings during competitions (Schade et al. 2004). Static calibration was performed using a $2 \times 2 \mathrm{~m}$ square and additional markers were placed at the level of the runway 4.5 and $5.5 \mathrm{~m}$ in front of the end of the planting box. The height of the center of the crossbar was included in the calibration process. The origin of the initial coordinate system was located above the deepest point of the planting box at ground level in the middle of the runway. The $x$-axis was defined as the horizontal axis in the main plane of movement. The $y$-axis was defined as the vertical axis. The digitization of body marks was performed using Skillspector ${ }^{\odot}$ software (Video4coach, Svendborg, Denmark) between the mid-flight before the last stance up to bar clearance. Next, the two-dimensional coordinates of the body landmarks were calculated from the digitized data using the two-dimensional direct linear transform (2D-DLT) algorithm.

\section{Body models of the athlete}

Three body models were used in this study (Figure 1). The first model contained 12 segments (reference model, $\mathrm{M}_{12}$ ) delineated by the following body markers: for both lower limbs the tip of the foot, ankle, knee, and hip joints; for both upper limbs the hand, elbow, and shoulder joints; and for the head C7-vertebrae and the middle of the head. The trunk was determined by both shoulders and both hips, whereas the hand and forearm were regarded as a single segment. An additional point not used in the reference model was digitized in the center of the pelvic girdle for construction of the other two models.

The second model contained five segments $\left(\mathrm{M}_{5}\right)$, whereas the third model contained three segments $\left(\mathrm{M}_{3}\right) . \mathrm{M}_{5}$ contained both upper limbs, both lower limbs, and one axial segment that encompassed the trunk and the head. Both upper limbs were delineated by the hand and C7vertebrae, and both lower limbs were delineated by the tip of the foot and the center of the pelvic girdle. The axial segment was delineated by the pelvic girdle marker and the middle of the head. $\mathrm{M}_{3}$ was defined similarly to $\mathrm{M}_{5}$ except that only the right upper limb (corresponding to the upper hand connected to the pole at take-off and the last hand to release the pole at the end of the vault) and the left lower limb (corresponding to the take-off foot) were retained.
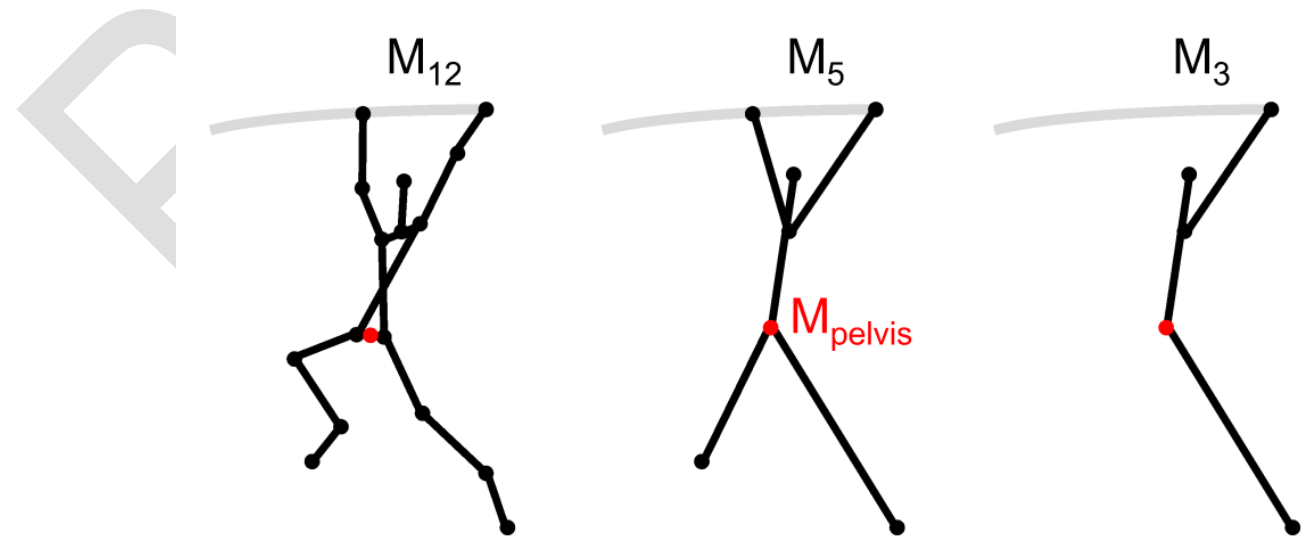

Figure 1. Visualization of the reference body model containing 12 segments $\left(M_{12}\right)$, and of the two simplified body models containing 5 and 3 segments $\left(M_{5}\right.$ and $M_{3}$, respectively). A marker located at the center of the pelvic girdle (red dot) was used to obtain a pseudo athlete's CoM ( $\left.\mathbf{M}_{\text {pelvis }}\right)$. 
The masses and moments of inertia of different segments were calculated using anthropometric data from Winter (2009), with relative masses adjusted by considering that $\mathrm{M}_{3}$ had one upper limb and one lower limb.

\section{Data processing}

Position data were smoothed using a Fourier series with an optimized order for the time-series of each point of the model (Jackson 1979). As done previously (Schade et al. 2000, 2004), two methods of mechanical energy calculation were used in this study. First, the athlete's total energy was calculated as follows:

$$
E_{\text {tot }}=\sum_{i=1}^{n} \frac{m_{i} v_{i}^{2}}{2}+\sum_{i=1}^{n} \frac{I_{i} \omega_{i}^{2}}{2}+\sum_{i=1}^{n} m_{i} g h_{i}
$$

where $m_{i}$ is the mass of the $i$ th segment, $v_{i}$ is the velocity of the CoM of the $i$ th segment, $\omega_{i}$ is the angular velocity of the $i$ th segment about the CoM of the body, $I_{i}$ is the moment of inertia of the $i$ th segment (de Leva 1996), $g$ is the acceleration of gravity $\left(g=9.81 \mathrm{~m} . \mathrm{s}^{-2}\right)$, and $h_{i}$ is the height of the CoM of the $i$ th segment. Equation 1 takes into account the linear and angular kinetic energy and potential energy of all segments in the studied model. Depending on the model, $n=12\left(\mathrm{M}_{12}\right), n=5\left(\mathrm{M}_{5}\right)$, or $n=3\left(\mathrm{M}_{3}\right)$.

Second, the athlete's CoM energy was calculated as follows:

$$
E_{C o M}=\frac{m v_{C o M}^{2}}{2}+m g h_{C o M}
$$

where $m$ is the mass of the athlete, $v_{C o M}$ is the velocity of the athlete's CoM, $g$ is the acceleration of gravity $\left(g=9.81 \mathrm{~m} \cdot \mathrm{s}^{-2}\right)$, and $h_{C o M}$ is the height of the athlete's CoM. Equation 2 takes into account the linear kinetic and potential energy of the athlete's CoM.

In addition to the six methods of energy computation (three body models and two approaches), we computed an additional pseudo athlete's CoM energy. Instead of using the kinematics of the athlete's CoM, we used Equation 2 with the kinematics of the point at the center of the pelvic girdle $\left(\mathrm{M}_{\text {pelvis }}\right.$; Figure 1). This choice was motivated by proposing a very economical approach but based on the abusive shortcut that assimilates the position of a point near the pelvis with the CoM of the body. For all computed energies, the values were normalized to the weight of the athletes to allow comparisons.

The energy time-profiles were normalized according to the instant of maximal pole bending (MPB) as described previously (Schade et al. 2000; Arampatzis et al. 2004). The bending phase between the touch-down of the last support and MPB was interpolated in order to obtain 100 data points. A similar process was performed for the straightening phase between MPB and the highest position of the athlete's CoM.

The primary energy parameters corresponded to the total or CoM energy of the vaulter at touch-down of the last support $\left(E_{\text {init }}\right)$, at the maximum pole bending position $\left(E_{M P B}\right)$, and at the maximum height of $\mathrm{CoM}\left(E_{H P}\right)$. Each of these three parameters was calculated by averaging three data points (i.e., the value before, at, and after the event). From these three primary energy parameters, three secondary parameters were obtained. The decrease in 
energy of the vaulter during the pole bending phase was the difference between the energy at touch-down and the energy at the maximum pole bending position $\left(E_{d e c r}=E_{\text {init }}-E_{M P B}\right)$. The increase in energy of the vaulter during the pole straightening phase was the difference between the energy at the maximum height of the CoM and the maximum pole bending position $\left(E_{\text {incr }}=E_{H P}-E_{M P B}\right)$. The energy gain $\left(E_{\text {gain }}=E_{H P}-E_{\text {init }}\right)$ was calculated from the difference between the energy of the vaulter at the maximum height of the CoM and the energy at touch-down (Schade et al. 2000; Frère et al. 2012).

\section{Statistical analysis}

Bland-Altman analysis was used to assess the agreement of each energy time-profile (linear and angular kinetic energy, potential energy, and mechanical energy) between the reference model $\left(\mathrm{M}_{12}\right)$ and the two other models $\left(\mathrm{M}_{5}\right.$ and $\left.\mathrm{M}_{3}\right)$. The linear, potential, and mechanical energy time-profiles obtained from $\mathbf{M}_{\text {pelvis }}$ were compared to those of $\mathrm{M}_{12}$ within the CoM approach. Changes in the bias (i.e., the mean difference) and $95 \%$ limits of agreement (i.e., standard deviation of the difference multiplied by 1.96) were assessed by paired student $t$-tests for the total approach and one-way ANOVA for the CoM approach.

Primary and secondary energy parameters were normally distributed (Shapiro-Wilk tests) with homogenous variances (two-sample $F$-tests). To assess the effect of the three models $\left(\mathrm{M}_{12}, \mathrm{M}_{5}\right.$, and $\left.\mathrm{M}_{3}\right)$ and the effect of calculation approaches (total vs. CoM energy) on the primary and secondary energy parameters, we used a two-way ANOVA for each parameter. A one-way ANOVA was used to assess the effect of the models $\left(\mathrm{M}_{12}, \mathrm{M}_{5}, \mathrm{M}_{3}\right.$, and $\left.\mathrm{M}_{\text {pelvis }}\right)$ on the energy parameters from the CoM approach. Post-hoc analyses were performed with Tukey/Kramer's tests. The level of significance was $p=0.05$.

\section{Results}

\section{Agreement of the energy time-profiles}

Overall, the energy time-profiles were highly similar among energy types (kinetic, potential, mechanical), computation approaches (total and CoM), and models $\left(\mathrm{M}_{5}, \mathrm{M}_{3}, \mathrm{M}_{\text {pelvis }}\right)$ in relation to the $M_{12}$ model (Table 1, Figure 2). For the total approach, $M_{12}$ vs. $M_{5}$ presented a higher absolute bias than $\mathrm{M}_{12}$ vs. $\mathrm{M}_{3}$ for the linear kinetic [t(19)=-12.3, $\left.p<0.001\right]$ and mechanical energy $[t(19)=-2.6, p<0.001]$. The opposite was found for the angular kinetic $[t(19)=12.4, p<0.001]$ and potential energy $[t(19)=-10.6, p<0.001]$. Limits of agreement were systematically lower between $\mathrm{M}_{12}$ and $\mathrm{M}_{5}$ than between $\mathrm{M}_{12}$ and $\mathrm{M}_{3}[t(19)$ ranging from -31.2 to $-12.7, p<0.001]$. For the CoM approach, we found a significant main effect for each energy type for the bias values $[F(2,57)$ ranging from 156.7 to $813.9, p<0.001]$ and the limits of agreement $[F(2,57)$ ranging from 295.4 to $762.4, p<0.001] . \mathrm{M}_{12}$ vs. $\mathrm{M}_{5}$ provided higher absolute bias for the linear kinetic and mechanical energy and lower absolute bias for potential energy than $\mathrm{M}_{12}$ vs. $\mathrm{M}_{3}$, and the $\mathrm{M}_{\text {pelvis }}$ model presented the highest absolute bias values with $\mathbf{M}_{12}$. For the total approach, limits of agreement between $\mathbf{M}_{12}$ and $\mathrm{M}_{5}$ were systematically the lowest when compared to the other simplified models. 
Table 1. Mean ( \pm SD) bias and limit of agreement in assessing the agreement in energy time-profiles between the models.

\begin{tabular}{|c|c|c|c|c|c|c|}
\hline & & \multicolumn{2}{|c|}{ Total approach } & \multicolumn{3}{|c|}{ CoM approach } \\
\hline & & $\mathrm{M}_{12}$ vs. $\mathrm{M}_{5}$ & $\mathrm{M}_{12}$ vs. $\mathrm{M}_{3}$ & $\mathrm{M}_{12}$ vs. $\mathrm{M}_{5}$ & $\mathrm{M}_{12}$ vs. $\mathrm{M}_{3}$ & $\mathrm{M}_{12}$ vs. $\mathrm{M}_{\text {pelvis }}$ \\
\hline \multirow[t]{4}{*}{$\begin{array}{r}\text { Bias } \\
\left(\mathrm{J.kg}^{-1}\right)\end{array}$} & $\begin{array}{l}\text { Linear } \\
\text { kinetic } \\
\text { energy }\end{array}$ & $-0.50( \pm 0.09)^{\mathrm{a}}$ & $-0.05( \pm 0.16)$ & $-0.51( \pm 0.07)^{\mathrm{a}, \mathrm{b}}$ & $-0.03( \pm 0.12)^{\mathrm{b}}$ & $-1.66( \pm 0.18)$ \\
\hline & $\begin{array}{r}\text { Angular } \\
\text { kinetic } \\
\text { energy }\end{array}$ & $-0.71( \pm 0.12)^{\mathrm{a}}$ & $-1.13( \pm 0.16)$ & - & - & - \\
\hline & $\begin{array}{r}\text { Potential } \\
\text { energy }\end{array}$ & $-0.04( \pm 0.03)^{\mathrm{a}}$ & $0.06( \pm 0.05)$ & $-0.04( \pm 0.03)^{\mathrm{a}, \mathrm{b}}$ & $0.06( \pm 0.05)^{\mathrm{b}}$ & $0.58( \pm 0.17)$ \\
\hline & $\begin{array}{r}\text { Mechanical } \\
\text { energy }\end{array}$ & $-1.25( \pm 0.17)^{\mathrm{a}}$ & $-1.12( \pm 0.22)$ & $-0.55( \pm 0.08)^{\mathrm{a}, \mathrm{b}}$ & $0.04( \pm 0.12)^{\mathrm{b}}$ & $-1.08( \pm 0.31)$ \\
\hline \multirow[t]{4}{*}{$\begin{array}{r}\text { Limit of } \\
\text { agreement } \\
\left({\left.\mathrm{J} . \mathrm{kg}^{-1}\right)}^{-1}\right.\end{array}$} & $\begin{array}{l}\text { Linear } \\
\text { kinetic } \\
\text { energy }\end{array}$ & $2.20( \pm 0.43)^{\mathrm{a}}$ & $8.28( \pm 0.96)$ & $2.01( \pm 0.37)^{\mathrm{a}, \mathrm{b}}$ & $=0.83)^{\mathrm{b}}$ & $3.19( \pm 0.95)$ \\
\hline & $\begin{array}{r}\text { Angular } \\
\text { kinetic } \\
\text { energy }\end{array}$ & $1.13( \pm 0.28)^{\mathrm{a}}$ & $2.17( \pm 0.33)$ & - & & - \\
\hline & $\begin{array}{r}\text { Potential } \\
\text { energy }\end{array}$ & $0.83( \pm 0.09)^{\mathrm{a}}$ & $1.23( \pm 0.14)$ & $0.83( \pm 0.09)^{\mathrm{a}, \mathrm{b}}$ & $1.23( \pm 0.14)^{b}$ & $2.6( \pm 0.20)$ \\
\hline & $\begin{array}{r}\text { Mechanical } \\
\text { energy }\end{array}$ & $2.51( \pm 0.68)^{\mathrm{a}}$ & $8.24( \pm 1.14)$ & $1.68( \pm 0.42)^{\mathrm{a}, \mathrm{b}}$ & $7.54( \pm 0.80)^{b}$ & $3.82( \pm 0.72)$ \\
\hline
\end{tabular}

${ }^{\mathrm{a}}$ significantly different from $\mathrm{M}_{12} v s . \mathrm{M}_{3} ;^{\mathrm{b}}$ significantly different from $\mathrm{M}_{12}$ vs. $\mathrm{M}_{\text {pelvis }}$.
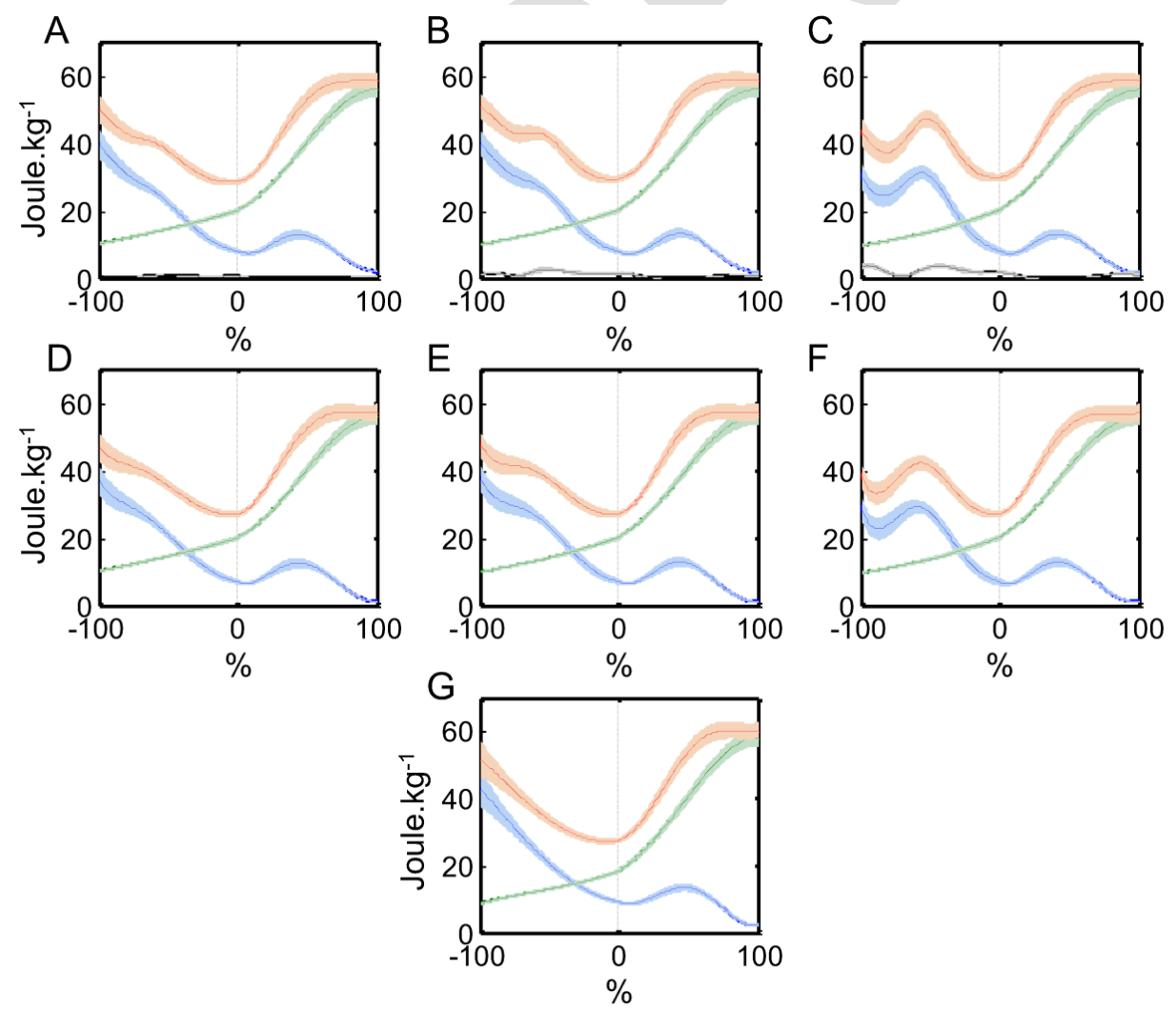

Figure 2. Mean ( \pm SD) normalized energy time-profiles during the pole vault from a total approach (A-C) and a CoM approach (D-E) using $M_{12}\left(A\right.$ and D), $M_{5}(B$ and $E), M_{3}(C$ and $F)$, and $M_{\text {pelvis }}(G)$. Blue, linear kinetic energy; black, angular kinetic energy; green, potential energy; red, athlete's mechanical energy. The $x$-axis is normalized as follows: $-100 \%$ up to $0 \%$ represents the phase between the beginning of the vault and MPB; $0 \%$ up to $+\mathbf{1 0 0 \%}$ represents the phase between the MPB and the highest position of the athlete's CoM. 


\section{Differences in energy parameters}

In regards to the primary parameters, we found a main effect of the models $[F(2,114)=52.2$, $p<0.001]$ and the computation approach $[F(1,114)=28.4, p<0.001]$ on the values of $E_{\text {init }}$, with no interaction effect $[F(2,114)=0.5, p=0.583]$. Post-hoc tests showed that $\mathrm{M}_{3}$ resulted in lower values of $E_{\text {init }}$ than $\mathrm{M}_{12}$ and $\mathrm{M}_{5}$, with no difference between $\mathrm{M}_{12}$ and $\mathrm{M}_{5}$ (Figure $3 \mathrm{~A}$ ). The total approach resulted in higher values of $E_{\text {init }}$ than the CoM approach. For $E_{M P B}$, we found a main effect of the models $[F(2,114)=4.5, p=0.013]$ and the computation approach $[F(1,114)=121.9$, $p<0.001]$, in addition to an interaction effect $[F(2,114)=3.5, p=0.034]$. Post-hoc tests showed that $\mathrm{M}_{3}$ had higher values of $E_{M P B}$ only with $\mathrm{M}_{12}$ (Figure $3 \mathrm{~B}$ ). The total approach resulted in higher values of $E_{M P B}$ than the CoM approach. The interaction effect could be understood as the model effect occurring solely for the total approach in energy computation. We found no main effect of the models on $E_{H P}[F(2,114)=0.1, p=0.867]$, though a main effect of the computation approach $[F(1,114)=13.8, p<0.001]$ was found, with the total approach giving higher values for $E_{H P}$ than the CoM approach (Figure 3C). We found no interaction effect $[F(2,114)=0.02, p=0.978]$ on $E_{H P}$.
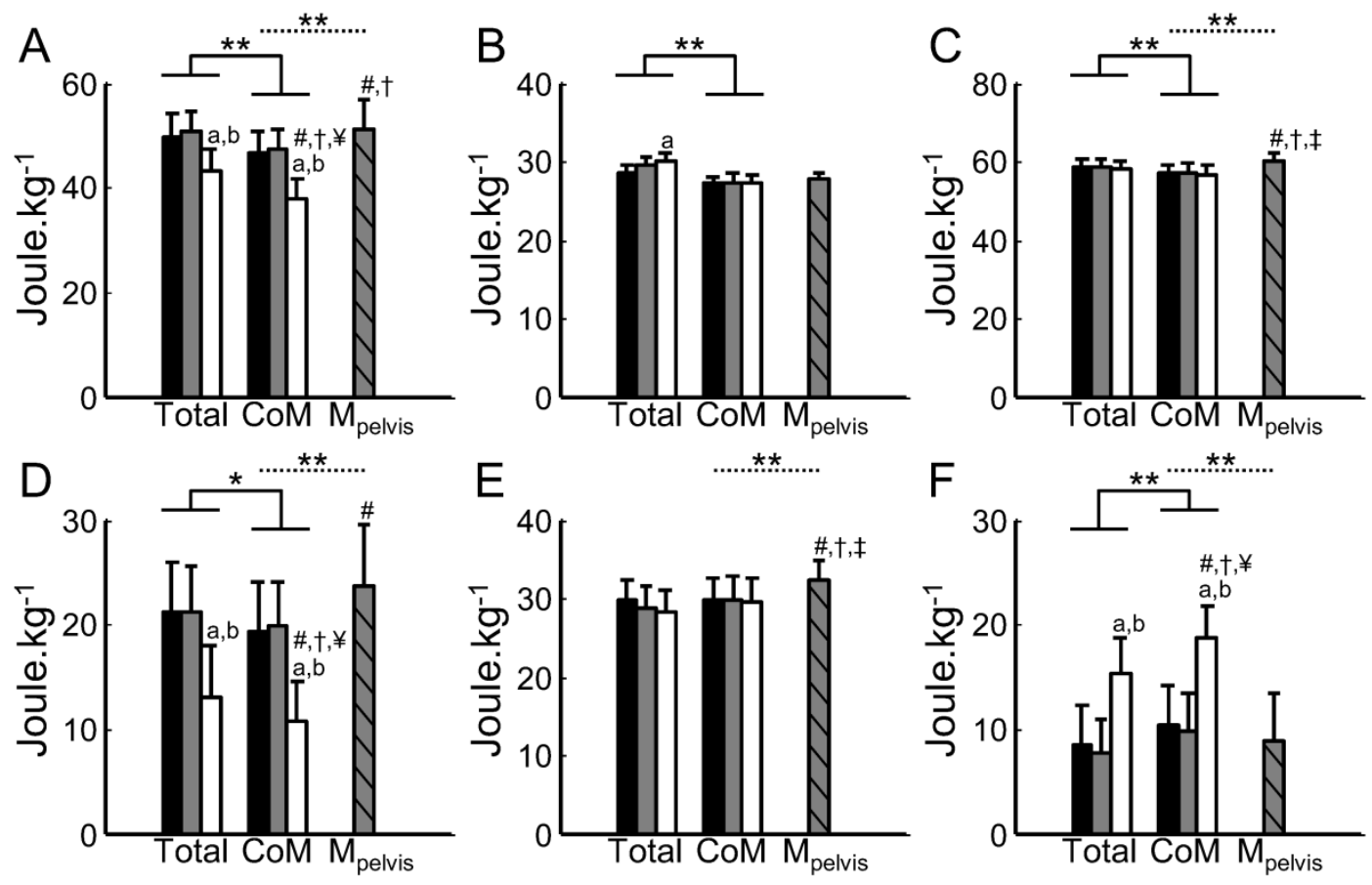

Figure 3. Differences in the primary $\left(E_{i n i t}, E_{M P B}\right.$, and $E_{H P}, \mathrm{~A}-\mathrm{C}$, respectively) and secondary $\left(E_{d e c}, E_{i n c r}\right.$, and $E_{\text {gain }}$, D-F, respectively) energy parameters among the studied models (black: $M_{12} ;$ grey: $M_{5}$; white: $\mathrm{M}_{3}$ ) and computation approaches (Total vs. CoM). ${ }^{*} \boldsymbol{p}<0.05, * *$ (continuous line) $p<0.01$ for ANOVA significant main effect of the energy computation approach. a: significantly different from $\mathrm{M}_{12} ; \mathrm{b}$ : significantly different from $M_{5}$ ** (dashed line) $p<0.01$ for ANOVA significant main effect of the body models $\left[M_{12}, M_{5}\right.$, and $M_{3}$ with the CoM approach $+M_{\text {pelvis }}$ (hatched bar)]. \# significantly different from $M_{12} ; \uparrow$ significantly different from $M_{5} ; \ddagger$ significantly different from $M_{3}$; $¥$ significantly different from $M_{\text {pelvis. }}$ Data are presented as mean \pm SD. 
Concerning the secondary parameters, we found a main effect of the models $[F(2,114)=49.0$, $p<0.001]$ and the computation approach $[F(1,114)=4.7, p=0.032]$ on the values of $E_{d e c r}$, with no interaction effect $[F(2,114)=0.1, p=0.863]$. Post-hoc tests showed that $\mathrm{M}_{3}$ resulted in lower values of $E_{\text {decr }}$ than $\mathrm{M}_{12}$ and $\mathrm{M}_{5}$, with no difference between $\mathrm{M}_{12}$ and $\mathrm{M}_{5}$ (Figure 3D). The total approach resulted in higher values of $E_{\text {decr }}$ than the CoM approach. For $E_{\text {incr }}$, we found no main effect of the models $[F(2,114)=0.8, p=0.431]$ or the computation approach $[F(1,114)=1.9, p=0.174]$, and no interaction effect $[F(2,114)=0.4, p=0.677]$ (Figure 3E). We found a main effect of the models $[F(2,114)=68.0, p<0.001]$ and the computation approach $[F(1,114)=15.4, p<0.001]$ on $E_{\text {gain }}$, with no interaction effect $[F(2,114)=0.6, p=0.547]$. Post$h o c$ tests showed that $\mathrm{M}_{3}$ resulted in higher values of $E_{\text {gain }}$ than $\mathrm{M}_{12}$ and $\mathrm{M}_{5}$ (Figure $3 \mathrm{~F}$ ), but we found no difference between $\mathrm{M}_{12}$ and $\mathrm{M}_{5}$. The total approach resulted in lower values of $E_{\text {gain }}$ than the CoM approach.

When comparing the primary and secondary energy parameters among the four models $\left(\mathrm{M}_{12}\right.$, $\mathrm{M}_{5}, \mathrm{M}_{3}$, and $\mathrm{M}_{\text {pelvis }}$ ) within the CoM approach, we found a main effect of the models on $E_{\text {init }}$ $[F(3,76)=33.1, p<0.001], E_{H P}[F(3,76)=9.6, p<0.001], E_{\text {decr }}[F(3,76)=27.4, p<0.001], E_{\text {incr }}$ $[F(1,114)=4.5, p=0.006]$, and $E_{\text {gain }}[F(3,76)=29.5, p<0.001]$, but we found no main effect of the models on $E_{M P B}[F(3,76)=1.0, p=0.420]$. Post-hoc tests showed that all pairwise comparisons were significant for $E_{\text {init }}$ and $E_{\text {decr }}$ except between $\mathrm{M}_{12}$ and $\mathrm{M}_{5}$ (for $E_{\text {init }}$ and $E_{\text {decr }}$, Figure $3 \mathrm{~A}$ ) and between $\mathrm{M}_{5}$ and $\mathrm{M}_{\text {pelvis }}$ (for $E_{\text {decr }}$ only, Figure $3 \mathrm{D}$ ). $\mathrm{M}_{\text {pelvis }}$ resulted in higher values of $E_{H P}$ (Figure 3C) and $E_{\text {incr }}$ (Figure 3E) than the other three models, but we found no difference among $\mathrm{M}_{12}, \mathrm{M}_{5}$, and $\mathrm{M}_{3}$. Finally, $E_{\text {gain }}$ was higher for $\mathrm{M}_{3}$ than $\mathrm{M}_{12}, \mathrm{M}_{5}$, and $\mathrm{M}_{\text {pelvis }}$ (Figure 3F).

\section{Discussion}

In this study, we analyzed the impact of simplifying the body model of the athlete on energy time-profiles and related parameters in pole vaulting. The main results were that reducing the body model had few effects on the energy time-profiles, except for the angular kinetic energy, but significant differences occurred in the primary and secondary energy parameters. Computing the total energy or the CoM energy, we found that $\mathrm{M}_{5}$ may be a good opportunity to simplify the body model without affecting outcomes related to the energy parameters.

In pole vaulting, the energy time-profiles are relevant to understanding the biomechanical aspects of the interaction between the athlete and the pole. Up to the time of maximal bending of the pole, the athlete's mechanical energy decreases because his horizontal displacement is slowed down by the force opposed by the pole, and the strain energy increases. When the pole is recoiling, the amount of strain energy decreases while the athlete's mechanical energy increases until the highest vertical position of his CoM (Arampatzis et al. 2004). Here, we found that regardless of the body model or computation approach, the energy time-profiles were similar to the reference model $\left(\mathrm{M}_{12}\right)$. These results agreed with those of Schade et al. (2000), who compared the total and CoM energy. This confirmed that a simple design for motion capture, even when tracking a unique point on the pelvis, allowed adequate determination of the changes in energies in pole vaulting. However, using $\mathrm{M}_{3}$ or $\mathrm{M}_{\text {pelvis }}$ resulted in decreased similarity to $\mathrm{M}_{12}$ in the energy time-profile, especially in the limit of 
agreement values, in contrast to the similarity between $\mathrm{M}_{12}$ and $\mathrm{M}_{5}$. For example, near half of the bending phase of the pole, the linear kinetic energy time-profile using $\mathrm{M}_{3}$ had a local peak (Figure $2 \mathrm{C}, 2 \mathrm{~F}$ ) that did not exist when using $\mathrm{M}_{12}$ or $\mathrm{M}_{5}$. This local peak with $\mathrm{M}_{3}$ could be explained by the body position at take-off, with the remaining hand and foot located behind the axial segment, leading to a backward shift of the body model's CoM. After holding this body position during a short period of time after take-off, the athlete initiated the swing movement, leading to a forward shift of the body model's CoM. By using a more complex model $\left(\mathrm{M}_{12}\right.$ or even $\left.\mathrm{M}_{5}\right)$, the $\mathrm{CoM}$ never had a backward position from the spine during and early after take-off. Therefore, the linear kinetic energy continuously decreased up to MPB (Dillman and Nelson 1968; Hay 1971; Gros and Kunkel 1990; Schade et al. 2000, 2004, 2006).

This change in linear and angular kinetic energies with the use of $\mathrm{M}_{3}$ had some consequences on the primary and secondary energy parameters. We found a higher $E_{\text {gain }}$ with $\mathrm{M}_{3}$ than with $\mathrm{M}_{12}$ or $\mathrm{M}_{5}$, which could be explained by the lower $E_{\text {init }}$ for $\mathrm{M}_{3}$, whereas we detected no effect of the model on $E_{H P}$. In addition, this lower $E_{\text {init }}$ for $\mathrm{M}_{3}$ resulted in a lower $E_{\text {decr }}$ compared to the other models, as a higher $E_{M P B}$ was found for $\mathrm{M}_{3}$. Overall, these results show that $\mathrm{M}_{3}$ seemed to not be a suitable body model for quantifying the energy parameters. Conversely, $\mathrm{M}_{5}$ did not have any significant difference from $\mathrm{M}_{12}$ for both primary and secondary energy parameters, in both total and CoM energy. Therefore, $\mathrm{M}_{5}$ seemed to be a good candidate for simplifying motion capture in pole vaulting. The five-segment model has several advantages: (i) it maintains the coarse configuration of the human body while the number of points to digitize is divided by more than 2; (ii) the remaining points to digitize are easy to track and visualize even in two dimensions, which is an advantage relative to the issue of marker occlusion; (iii) it preserves the opportunity to compute the strain energy stored in the pole, in addition to recording the kinetic data, due to the compression force and bending torque applied by both hands of the athlete as proposed by Arampatzis et al. (2004). Furthermore, in agreement with Schade et al. (2000), we found that the total energy approach generally resulted in higher energy parameter values, regardless of the body model used. Notably, $E_{M P B}$ was the sole energy parameter to present an interaction effect between the models and the computation approaches. Though the models did not influence $E_{M P B}$ with the CoM approach, the simplification of the body model increased $E_{M P B}$ with the total approach. Due to the increasing preponderance of the remaining segments, the amount of angular kinetic energy increased with simplification of the model and resulted in higher mechanical energy at MPB. Overall, this model effect underlined the substantial role of the gestural technique of the athlete, especially the swing-to-inversion movement performed during the bending phase of the pole, though only with the total approach (Hay 1971; Hubbard 1980; Ekevad and Lundberg 1995, 1997; Arampatzis et al. 2004; Frère et al. 2012).

Surprisingly, the most economical approach $\left(\mathrm{M}_{\text {pelvis }}\right)$ provided quite fair results for all of the studied aspects. The agreement of the energy time-profiles with $\mathrm{M}_{12}$ was slightly less than that of $\mathrm{M}_{5}$ and slightly more than that of $\mathrm{M}_{3}$ (Table 1), whereas some energy parameters were not significantly different from those obtained by $\mathrm{M}_{12}$ or $\mathrm{M}_{5}$. Notably, if we solely focus on $E_{\text {gain }}$, $\mathrm{M}_{\text {pelvis }}$ appeared to be a suitable method, but $\mathrm{M}_{\text {pelvis }}$ tended to overestimate almost all of the 
other primary and secondary energy parameters, which reflects the misconception in confounding the kinematics of a pelvis marker with those of the body model's CoM.

One can argue that a way to decrease the cost of data processing without simplifying the body model could be to select only the frames of interest instead of continuously capturing the whole movement. This previously used method (Arampatzis et al. 1999) is interesting because it presents the main advantage of drastically decreasing the number of remaining frames to digitize ( 20 instead of $>200)$ while retaining a standard full body model, but this is not without limitations. First, the smoothing of the data may be an issue when keeping a limited number of frames per event, and even more so in a context of manual digitization. This may also bring into question the reliability of the results, especially for the calculation of velocities. Second, some events are not always easy to detect a priori by video visualization. For example, the highest vertical position of the CoM can be detected, by definition, only after the digitization process allowing calculating of the position of CoM. This is also true for the MPB. Third, with such a method, we focus solely on the primary and secondary energy parameters of the pole vault studied. Even if these parameters could be considered to be highly relevant in the field, the literature related to pole vaulting is still relatively sparse. Therefore, one can easily imagine that other energy-based performance factors could be determined in future studies.

In conclusion, the five-segment model appeared to be a good compromise between decreasing the number of points to digitize and maintaining the reliability of outcomes in terms of energy time-profiles and energy parameters. Therefore, such a simple body model creates the possibility of quickly providing objective and quantified feedback to coaches and athletes (using $\mathrm{M}_{5}$ allowed to digitize the whole movement in 15-20 minutes instead of more than one hour with $\mathrm{M}_{12}$ ), which could be of interest for skill learning, decision-making, training programs, or pole selection.

Funding: This work was supported by the French Ministry of Sport and the French Institute of Sport under Grant 16-R-14, and the French Athletics Federation.

Disclosure statement: No potential conflict of interest was reported by the authors.

\section{References}

Abdollahipour R, Wulf G, Psotta R, Palomo Nieto M. 2015. Performance of gymnastics skill benefits from an external focus of attention. J Sports Sci. 33:1807-1813.

Adamczewski H, Perlt B. 1997. Run-up velocities of female and male pole vaulting and some technical aspects of women's pole vault. New Stud Athl. 12:63-76.

Arampatzis A, Schade F, Brüggemann G-P. 1999. Pole vault. In: Brüggemann G-P, Koszewski D, Müller H, editors. Biomech Res Proj VIth World Championships Athl Athens 1997 Final Rep. Oxford: Meyer \& Meyer Sport; p. 145-160.

Arampatzis A, Schade F, Brüggemann G-P. 2004. Effect of the pole--human body interaction on pole vaulting performance. J Biomech. 37:1353-1360. 
Dillman CJ, Nelson RC. 1968. The mechanical energy transformations of pole vaulting with a fiberglass pole. J Biomech. 1:175-183.

Ekevad M, Lundberg B. 1995. Simulation of “smart” pole vaulting. J Biomech. 28:1079-1090.

Ekevad M, Lundberg B. 1997. Influence of pole length and stiffness on the energy conversion in polevaulting. J Biomech. 30:259-264.

Frère J, Göpfert B, Slawinski J, Tourny-chollet C. 2012. Effect of the upper limbs muscles activity on the mechanical energy gain in pole vaulting. J Electromyogr Kinesiol Off J Int Soc Electrophysiol Kinesiol. 22:207-214.

Frère J, L'Hermette M, Slawinski J, Tourny-Chollet C. 2010. Mechanics of pole vaulting: a review. Sports Biomech Int Soc Biomech Sports. 9:123-138.

Gros H, Kunkel V. 1990. Biomechanical analysis of the pole vault. In: Brüggemann G-P, Glad B, editors. Sci Res Proj Games 24th Olymp - Seoul 1988 Final Rep. Monaco: International Amateur Athletic Federation; p. 219-260.

Hay JG. 1971. Mechanical energy relationships in vaulting with a fibreglass pole. Ergonomics. 14:437-448.

Hubbard M. 1980. Dynamics of the pole vault. J Biomech. 13:965-976.

Jackson KM. 1979. Fitting of mathematical functions to biomechanical data. IEEE Trans Biomed Eng. 26:122-124.

de Leva P. 1996. Adjustments to Zatsiorsky-Seluyanov's segment inertia parameters. J Biomech. 29:1223-1230.

Marchant DC, Greig M, Scott C. 2009. Attentional focusing instructions influence force production and muscular activity during isokinetic elbow flexions. J Strength Cond Res. 23:2358-2366.

Schade F, Arampatzis A, Brüggemann G. 2000. Influence of different approaches for calculating the athlete's mechanical energy on energetic parameters in the pole vault. J Biomech. 33:1263-1268.

Schade F, Arampatzis A, Brüggemann G-P. 2006. Reproducibility of energy parameters in the pole vault. J Biomech. 39:1464-1471.

Schade F, Arampatzis A, Brüggemann G-P, Komi P. 2004. Comparison of the men's and the women's pole vault at the 2000 Sydney Olympic Games. J Sports Sci. 22:835-842.

Winter DA. 2009. Biomechanics and motor control of human movement. Fourth Edition. Hoboken, New Jersey: John Wiley \& Sons, Inc.

Wulf G, Chiviacowsky S, Schiller E, Avila LTG. 2010. Frequent external-focus feedback enhances motor learning. Front Psychol. 1:190.

Wulf G, Dufek JS, Lozano L, Pettigrew C. 2010. Increased jump height and reduced EMG activity with an external focus. Hum Mov Sci. 29:440-448.

Wulf G, McNevin N, Shea CH. 2001. The automaticity of complex motor skill learning as a function of attentional focus. Q J Exp Psychol A. 54:1143-1154. 